\title{
Measurement of anisotropies in cosmic ray arrival directions with the Alpha Magnetic Spectrometer on the ISS
}

\author{
Iris Gebauer* on Behalf of the AMS Collaboration \\ Karlsruhe Institute of Technology, Institute for Experimental Particle Physics, D-76131 \\ Karlsruhe, Germany \\ E-mail: gebauer@kit.edu

\section{F. Bindel, M. Graziani, S. Zeissler} \\ Karlsruhe Institute of Technology, Institute for Experimental Particle Physics, D-76131 \\ Karlsruhe, Germany
}

\section{J. Casaus, C. Mana, M. A. Velasco}

Centro de Investigaciones Energéticas, Medioambientales y Tecnológicas (CIEMAT), E-28040

Madrid, Spain

\section{Gervasi, G. La Vacca, P.G. Rancointa}

INFN Sezione di Milano-Bicocca, I-20126 Milano, Italy

\begin{abstract}
An analysis of anisotropies in the arrival directions of galactic protons, electrons and positrons has been performed with the Alpha Magnetic Spectrometer on the International Space Station using the first five years of data taking. Results on a dipole signal in Galactic coordinates are reported. The positron to proton and positron to electron ratio is consistent with isotropy. For energies above $16 \mathrm{GeV}$ a limit of $\delta<0.02$ at the $95 \%$ confidence level is obtained.

The electron to proton ratio is consistent with isotropy. For energies above $16 \mathrm{GeV}$ a limit of $\delta<0.004$ at the $95 \%$ confidence level is obtained.

The ratio of high rigidity protons to low rigidity protons is consistent with isotropy. For energies above $80 \mathrm{GV}$ a limit of $\delta<0.002$ at the $95 \%$ confidence level is obtained.

Limits on the absolute anisotropies in protons, electrons and positrons are consistent with the limits derived from the relative anisotropies. No indication of any time dependence is observed for all particle species within the present statistics.
\end{abstract}

35th International Cosmic Ray Conference - ICRC2017

10-20 July, 2017

Bexco, Busan, Korea

\footnotetext{
* Speaker.
} 


\section{Introduction}

The precise measurements of Cosmic Ray (CR) fluxes performed by the Alpha Magnetic Spectrometer (AMS-02) onboard the ISS, have revealed multiple unexpected features which cannot be explained in the standard paradigm of cosmic ray transport: for example, the spectra of protons and helium exhibit a hardening at rigidities of about $300 \mathrm{GV}$ [1,2], which might be caused by the presence of a local CR accelerator, which dominates the local CR spectra above a few hundred GV. Further, the positron fraction shows a rise above a few $\mathrm{GeV}$ with a slope decreasing logarithmically with energy above $30 \mathrm{GeV}$ and above $200 \mathrm{GeV}$ the positron fraction is no longer increasing with energy [3]. This behaviour originates from the electron and positron fluxes dependence on energy. Above around $20 \mathrm{GeV}$ and up to $200 \mathrm{GeV}$ the electron flux decreases more rapidly with energy than the positron flux, that is, the electron flux is softer than the positron flux [4].

These features cannot be explained within our current understanding of galactic cosmic ray transport. They may be related to the presence of unaccounted astrophysical sources, e.g. local supernova remnants or Wolf-Rayet stars might be the source of the hard proton and helium component, which dominates the respective $\mathrm{CR}$ spectra above a few hundreds of $\mathrm{GV}$, pulsars might produce energetic electron-positron pairs in their strong rotating magnetic fields, which are able to explain the observed rise and flattening on the positron fraction.

If the observed features are indeed caused by a limited number of astrophysical point sources, such a point source needs to be relatively close by. Otherwise fast energy losses for electrons and positrons or escape from the galaxy for protons and healium would significantly soften the CR spectra. A limited number of close-by point sources could induce some degree of anisotropy in the $\mathrm{CR}$ arrival directions. Therefore it is worth to measure and characterize the angular distribution of CR arrival directions. Anisotropy searches therefore provide complementary information to constrain the origin of the observed features and the propagation and production mechansims of CRs in the Galaxy.

In this study we analized a sample of protons, electrons and positrons taken in the first five years of AMS-02 data taking between $18 \mathrm{GV}$ and $1.8 \mathrm{TV}$ for protons and $16 \mathrm{GeV}$ and $350 \mathrm{GeV}$ for electrons and positrons. A search for relative and absolute anisotropies in the arrival directions of cosmic rays and a systematic study of the seasonal dependence of the anisotropy was performed.

\section{The AMS-02 Detector}

AMS-02 is a large acceptance multipurpose particle detector, designed to measure the fluxes of individual cosmic ray species at $\mathrm{GeV}$ and $\mathrm{TeV}$ energies. It was installed on 19 May 2011 onboard the International Space Station (ISS) and has been continuously taking data since then, collecting more than 100 billion events of galactic CRs. The detector will remain on the ISS and continue to take data until the end of ISS operation which is currently planned for 2024. The detector consists of nine precision silicon tracker planes with two outer planes, 1 and 9 , and the inner tracker, planes 2 to 8 ; a transition radiation detector (TRD); four planes of time of flight (TOF) counters; a permanent magnet; an array of anticoincidence counters (ACC), inside the magnet bore; a ring imaging Čerenkov detector (RICH); and an electromagnetic calorimeter (ECAL). A detailed dectector description can also be found in $[1,2,3,4,5,6]$. 


\section{Data Selection}

Anisotropy searches rely on directional information for single events. To preserve single particle information a cut based electron and positron selection is performed. The basic quality selection follows the description in [4] and in [3], but no template fits in ECAL and TRD estimators are performed to identify particles. The reduction of the proton background in the identification of the positron and electron samples is achieved by means of the TRD, the ECAL and the tracker. Events are selected by requiring a minimum of 8 TRD hits consistent with the tracker track, a cluster of hits in the ECAL, and a measured velocity $\beta \sim 1$ in the TOF consistent with a downward-going $\mathrm{Z}=1$ particle. Protons are rejected by requiring a good energy-momentum matching and explicit cuts on the ECAL and TRD estimators. The overall selection efficiency for positrons and electrons is estimated to be above $80 \%$ compared to the template fit analysis in [4] and [3]. The remaining sample contains 70000 positrons, 920000 electrons above $16 \mathrm{GeV}$ with negligible proton contamination. The selected events are grouped into 5 cumulative energy ranges from 16 to $350 \mathrm{GeV}$ according to their measured energy in the ECAL. The minimum energies are 16, 25, 40, 65 and $100 \mathrm{GeV}$, respectively.

The proton selection follows the one described in [1]. The first step of the selection requires the preselection of events in which the velocity was measured by four TOF layers being consistent with down-going particles. Further cuts are made requiring the charge consistency with $\mathrm{Z}=1$ particle and requiring the track, reconstructed by the 7 inner layers, to pass through the outer layers 1 and 9 and to satisfy additional track fitting quality criteria.

In addition, to select only primary CRs, well above the geomagnetic cutoff, the measured rigidity is required to be greater than 1.2 times the maximum geomagnetic cutoff within the AMS field of view.

\section{Method}

The ISS orbit inclination of $51.6^{\circ}$ relative to the Earth's equator leads to a non-uniform sky coverage of the AMS-02 detector. The left side of Fig. 1 shows the AMS-02 pointing direction over a period of 2.5 years in galactic coordinates. Regions with high exposure around the Earth's north and south pole are clearly visible. The finite detector acceptance of about $35^{\circ}$ for a proton selection and $25^{\circ}$ for an electron selection, smears out the pointing direction, leading to rings of high exposure around the Earth's north and south poles. The right side of Fig. 1 shows the proton sky above $40 \mathrm{GV}$ as seen by AMS-02. The nearly full sky covererage allows to search for a full 3-dimensional dipole in CR arrival directions.

The CR arrival directions are binned in sky maps in galactic coordinates $(l, b)$ containing the number of observed particles using the HEALPix ${ }^{1}$ pixelization, which guarantees a regular sampling of the $\mathrm{CR}$ angular distribution.

The efficiency with wich particles can be detected by AMS-02 depends on the trigger rate which changes with geomagnetic latitude as a result of the bending of the Earth's magnetic field lines. Towards the poles a higher rate of low rigidity particles is able to reach ISS orbit, while

\footnotetext{
${ }^{1} \mathrm{http} / / /$ healpix.sourceforge.net
} 

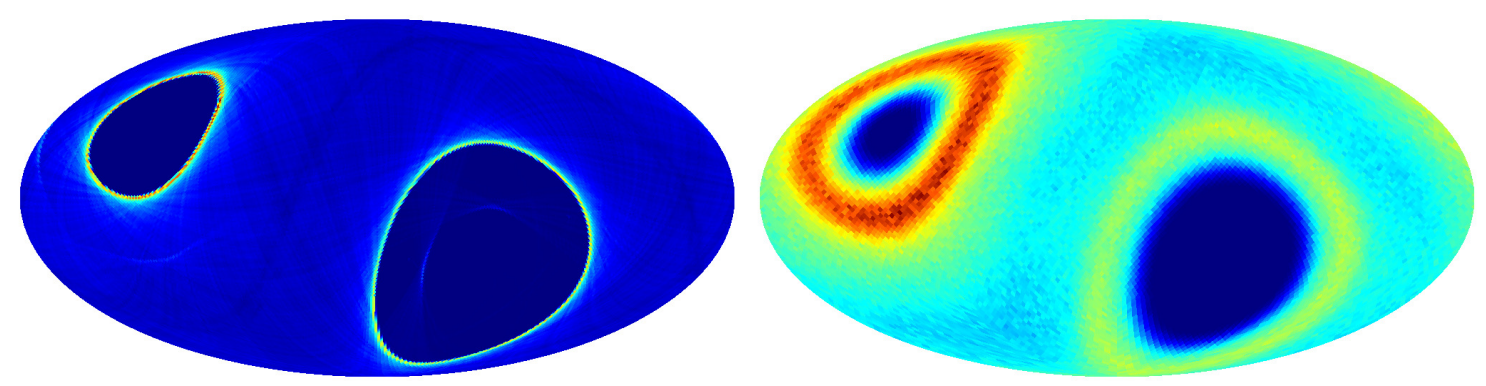

Figure 1: Left: The AMS-02 pointing direction over a period of 2.5 years. The color coding illustrates the number of seconds the z-axis of the detector spends pointing to a vertain position in the sky. Right: The proton sky above $40 \mathrm{GeV}$ as observed by AMS-02.

at the equator these particles are reflected by the Earth's magnetic field. In addition, single cut efficiencies may have a dependence on detector position.

Above $16 \mathrm{GeV}$ all $\mathrm{CR}$ species share the same exposure ${ }^{2}$, while selection efficiencies and acceptance may be different as a function of detector position.

The search for anisotropies makes it necessary to first estimate an accurate representation of how an isotropic sky would look like for any given particle selection. For this so-called reference maps are used. A reference map is any map providing our best guess of what an isotropic sky would look like for the respective CR species, collected by the same detector in the same datataking period. Any deviation between the reference map and the data might be detected as a signal. Here we present results for relative anisotropies using protons as a reference maps for electrons, electrons and protons as a reference map for positrons, protons at low rigidities as a reference for protons at high rigidities, as well as so-called IsoSky maps simulated from data of the same CR species.

Using a likelihood fit proceedure, the normalized ratio of data map and reference map is expanded into spherical harmonics:

$$
\phi_{i}=\sum_{\ell=0}^{\ell_{\max }} \sum_{m=-\ell}^{\ell} a_{\ell m} Y_{\ell m}\left(l_{i}, b_{i}\right),
$$

where $\left(l_{i}, b_{i}\right)$ is the position of the $i^{\text {th }}$ pixel in galactic coordinates. The dipole for $\ell=1$ is fully described by three orthogonal functions aligned in galactic coordinates with directions: $Y_{10}$ along the North-South (NS) direction perpendicular to the galactic plane, $Y_{11}$ along the Forward-Backward (FB) direction with respect to the galactic center and $Y_{1-1}$ along the East-West (EW) direction tangent to the orbit of the sun around the galactic center. It is then worth to study these three directions both separately and combined to determine the total omnidirectional dipole magnitude

$$
\delta=\sqrt{\rho_{N S}^{2}+\rho_{F B}^{2}+\rho_{E W}^{2}} .
$$

where the three dipole coefficients are defined as

$$
\rho_{N S}=\sqrt{\frac{3}{4 \pi}} a_{10}, \quad \rho_{F B}=\sqrt{\frac{3}{4 \pi}} a_{11}, \quad \rho_{E W}=\sqrt{\frac{3}{4 \pi}} a_{1-1} .
$$

This procedure proved to be stable against different map resolutions and sample statistics.

\footnotetext{
${ }^{2}$ below $16 \mathrm{GeV}$ the East-West effect may induce differences in the exposure of particle of different charges
} 

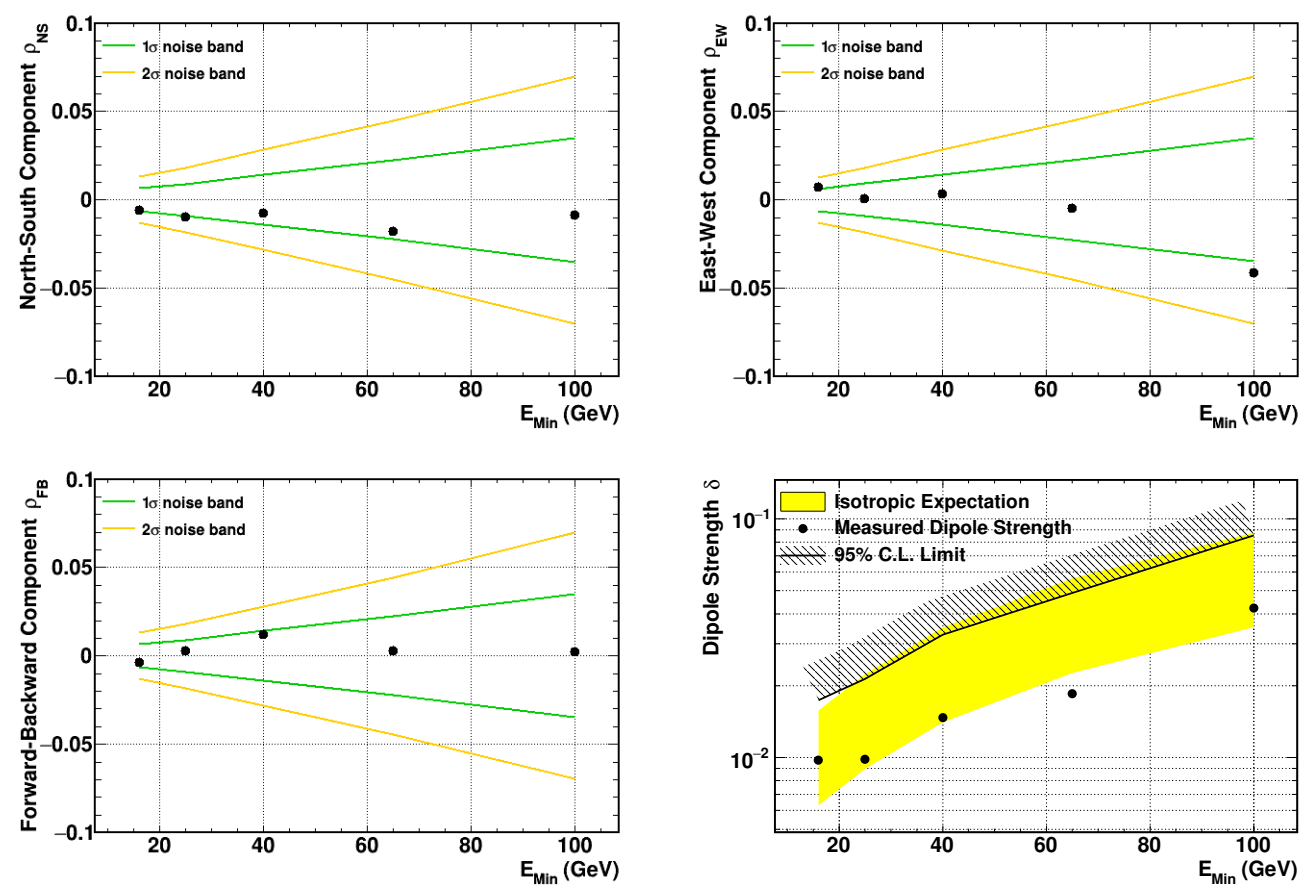

Figure 2: Amplitudes of the $\rho_{N S}$ (top left), $\rho_{E W}$ (top right) and $\rho_{F B}$ bottom left dipole components obtained for different energy ranges in the positron over proton ratio. Bottom right: The 95\% C.L. upper limit obtained from the dipole fits on the other panels. The upper limit is calculated from monte carlo using simulated dipoles and isotropic reference maps. A bayesian 95\% C.L. is computed from the distribution of true dipole strengths for the dipole strength measured on data. Also shown is the isotropic expectation calculated from simulated isotropic signal and reference maps corresponding to the respective positron and protons statistics. The isotropic expectation is quoted as the $68.3 \%$ C.L. of the reconstructed $\delta$ distribution.

\section{Anisotropy in positron to proton and positron to electron ratio}

In Fig. 2 the dipole components for the positron to proton ratio are shown as function of the minimum energy. No significant deviation from isotropy can be observed. The corresponding $95 \%$ C.L. upper limits on the dipole amplitude for different energies are reported on the right side of the bottom panel of Fig. 2. The limit obtained for the energy range from 16 to $350 \mathrm{GeV}$ is $\delta<0.02$.

To check for a possible time dependence of the reconstructed dipole components the full datataking period was divided into 20 seasons and the analysis is repeated on the individual samples. No significant time dependence was observed.

To check for possible systematics from the proton reference map, which might be caused by different dependencies of the proton and positron selection efficiencies as a function of geomagnetic position, the analysis was repeated using electrons as a reference sample. Also here no significant devciation from isotropy and no time dependence in the dipole components was found. The limit obtained for the energy range from 16 to $350 \mathrm{GeV}$ is $\delta<0.02$. This limit is consistent with the one reported in [5], the only improvement due to the increased statistics after five years.

A similar search for anisotropies in the electron to proton ratio was performed. No significant deviation from isotropy and no significant time dependence was observed. A limit of $\delta<0.004$ at 

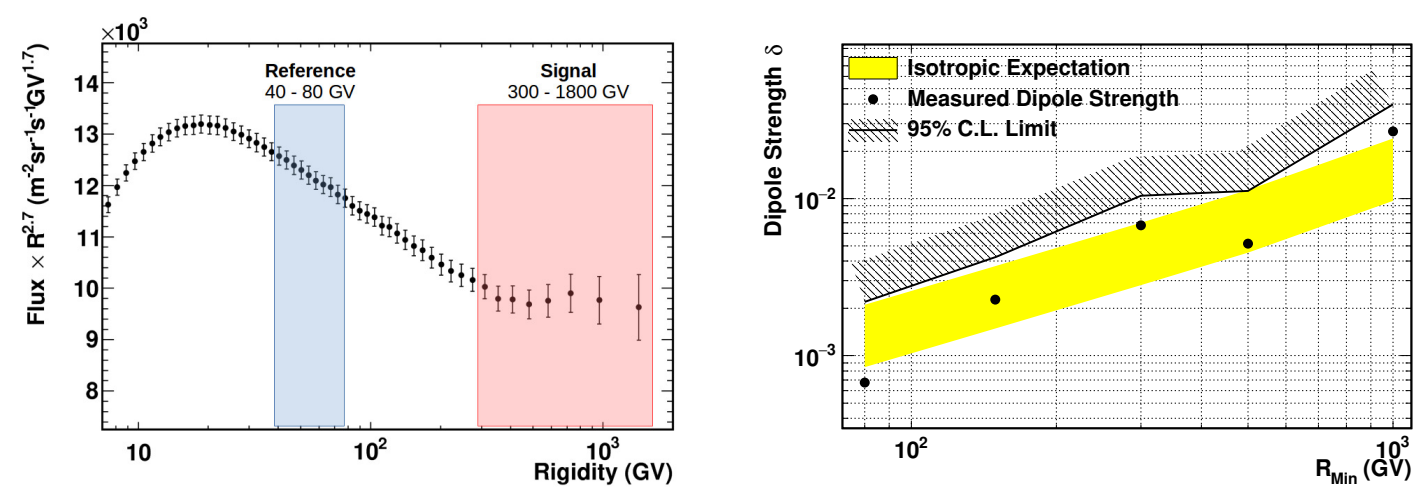

Figure 3: Left: The choice of reference sample for the study of anisotropies in high energy protons. A reference sample between $[40,80] \mathrm{GV}$ is chosen and compared to the signal sample at higher rigidities. Right: Upper limits at 95\% C.L. of the omnidirectional dipole intensity in the high rigidity proton to low rigidity proton ratio.

the $95 \%$ C.L. was found for energies between 16 and $350 \mathrm{GeV}$.

\section{Anisotropy in high energy protons}

For protons no other CR species can serve as a reference, since no species is more abundant in $\mathrm{CR}$ than protons. Using a less abundant species as reference would make the statistics of the normalized ratio entering the analysis as described in section 4 dominated by statistical fluctuations of the reference sample. To search for a possible anisotropy in the arrival directions of protons at high rigidities, which is possibly associated to the origin of the change of slope in the proton spectrum at around $300 \mathrm{GV}$, a different ansatz is chosen: a sky map of protons in the range [40,80] GV, well above the geomagnetic cutoff, is used as reference map for higher rigity protons. For this analysis protons are grouped into 5 cumulative rigidity bins from 80 to $1800 \mathrm{GV}$ according to their measured rigidity. The minimum rigidities are $80,150,300,500,1000 \mathrm{GV}$, respectively. The left side of Fig. 3 illustrates our choice of reference sample, while the right side shows the 95\% C.L. upper limits on the dipole amplitude for different minimium rigidities. The points at every rigidity show no significant deviation from isotropy. The limit obtained for the rigidity range from 80 to $1800 \mathrm{GV}$ is $\delta<0.002$. Also in this case no seasonal effects are observed.

\section{Absolute anisotropies in protons, electrons and positrons}

Relative anisotropies are convenient, since suitable reference maps for most species are readily available. Even if the reference map might carry some of the signal expected in the signal map, like in the case of positrons and electrons where a pulsar contribution is expected for both particle species, a meaningful model comparison can still be performed by calculating the expected relative model anisotropy. However, each species undergoes different selection efficiencies and detector systematics. As long as these effects do not change differently as a function of position for the signal and reference species, no impact on the final result is expected. For protons and electrons, however, the selection efficiencies can vary as a function of detector position, due to the 
different impact of the Earth's magnetic field on different selection cuts. Likewise for positrons and electrons, a difference might be due to the different propagation of opposite electric charges in the Earth's magnetic field. To avoid systematic effects introducing fake signals or hiding possible physical signals, a method to calculate absolute anisotropies from a simulated isotropic reference map was derived. The method was introduced in [7]. It projects the detector's field of view into the galactic sky and then weights the accumulated livetime in this field of view with the detector efficiencies for the respective particle selection according to the following algorithm: Start by getting a list of particle incoming directions $(\theta, \phi)$ in detector coordinates from the selected data events. Then, for every second of data taking:

1. Draw $\mathbf{N}$ sets of incoming directions $(\theta, \phi)_{i}$ from this list. (Geometric acceptance is preserved.)

2. Calculate the galactic arrival direction $\Psi_{i}$ from the drawn incoming directions and the detector position in the respective second. (The geometric acceptance is projected onto the galactic sky.)

3. Weight the events with the detector livetime $T_{\exp }$ in this second to account for busy trigger and a time and position dependent correction factor $\varepsilon(t, \vec{x})$, to account for time and position dependent efficiencies.

An example of a resulting reference map, called IsoSky map, for protons can be found in the top left of Fig. 4. The method was applied to protons, electrons and positrons. No significant deviation from isotropy was found for any CR species. No significant time dependence was observed. The 95\% C.L. upper limits obtained are shown in Fig. 4 together with the isotropic expectation for galactic coordinates. For protons an upper limit of $\delta<0.001$ above $18 \mathrm{GV}$ was found, for electrons and positrons an upper limit of $\delta<0.004$ and $\delta<0.02$, respectively, above $16 \mathrm{GeV}$ was found.

\section{Conclusions}

A search for a dipole signal in the arrival directions of protons, electrons and positrons was performed using 5 years of data taken with the AMS-02 detector onboard the ISS. No significant deviation from isotropy was observed, no significant time dependence was found within the current statistics. A $95 \%$ confidence level upper limit of $\delta<0.02$ and $\delta<0.004$ was obtained for the energy range 16 to $350 \mathrm{GeV}$ for positrons and electrons, respectively. For protons a $95 \%$ confidence level upper limit of $\delta<0.001$ was obtained for the rigidity range from 18 to $1800 \mathrm{GV}$. Each analysis was performed using two independent references maps, derived either directly from CRs or from IsoSky maps. The obtained results are consistent.

\section{Acknowledgments}

This work has been supported by the persons and institutions acknowledged in [2]. Some of the results in this paper have been derived using the HEALPix (K.M. Górski et al., 2005, ApJ, 622, p759) package. 

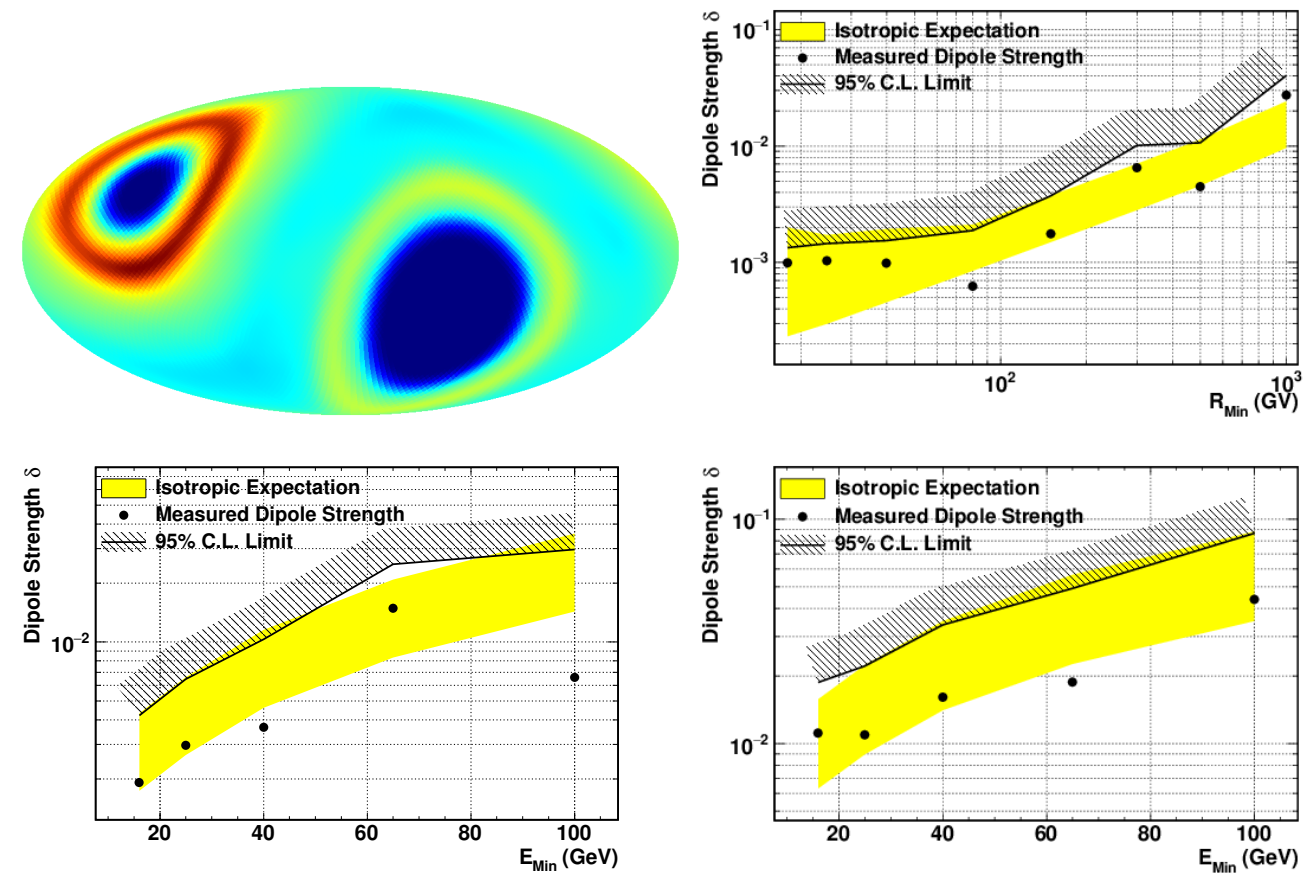

Figure 4: Top left: IsoSky map for protons above $40 \mathrm{GV}$ in galactic coordinates. Other figures: the $95 \%$ C.L. upper limits for the omnidirectional dipole intensity for absolute protons (top right), absolute electrons (bottom left) and absolute positrons (bottom right).

\section{References}

[1] M. Aguilar et al., "Precision measurement of the proton flux in primary cosmic rays from rigidity 1 gv to $1.8 \mathrm{tv}$ with the alpha magnetic spectrometer on the international space station," Phys. Rev. Lett., vol. 114, p. 171103, Apr 2015.

[2] M. Aguilar et al., "Precision measurement of the helium flux in primary cosmic rays of rigidities $1.9 \mathrm{gv}$ to $3 \mathrm{tv}$ with the alpha magnetic spectrometer on the international space station," Phys. Rev. Lett., vol. 115, p. 211101, Nov 2015.

[3] L. Accardo et al., "High statistics measurement of the positron fraction in primary cosmic rays of 0.5-500 gev with the alpha magnetic spectrometer on the international space station," Phys. Rev. Lett., vol. 113, p. 121101, Sep 2014.

[4] M. Aguilar et al., "Electron and positron fluxes in primary cosmic rays measured with the alpha magnetic spectrometer on the international space station," Phys. Rev. Lett., vol. 113, p. 121102, Sep 2014.

[5] M. Aguilar et al., "First result from the alpha magnetic spectrometer on the international space station: Precision measurement of the positron fraction in primary cosmic rays of 0.5-350 gev," Phys. Rev. Lett., vol. 110, p. 141102, Apr 2013.

[6] M. Aguilar et al., "Precision measurement of the $\left(e^{+}+e^{-}\right)$flux in primary cosmic rays from 0.5 gev to 1 tev with the alpha magnetic spectrometer on the international space station," Phys. Rev. Lett., vol. 113, p. 221102, Nov 2014.

[7] I. Gebauer, "Direction and time dependent fluxes with AMS-02," PoS, vol. ICRC2015, p. 404, 2016. 